\title{
Erratum to "Hypersurfaces of constant mean curvature with finite index and volume of polynomial growth"
}

\author{
By
}

H. ALENCAR and M. Do Carmo

The proof of Lemma (2.4) in our paper [1] is incorrect. We have to change slightly the notion of polynomial growth and say that volume of $M$ has polynomial growth if there exist positive members $\alpha$ and $c$ such that

$$
\limsup _{r \rightarrow \infty} \frac{V(B(r))}{r^{\alpha}}=c
$$

Now replace p. 492 line 11 up to p. 493 line 4 by the following:

To prove this, we notice that the fact that the volume has polynomial growth implies that we can choose an increasing sequence $\left\{q_{i}\right\}$ of radi, $q_{i} \rightarrow \infty$, such that

$$
\lim _{q_{i} \rightarrow \infty} \frac{V\left(B\left(q_{i}\right)\right)}{q_{i}^{\alpha}}=c .
$$

Now consider the sequence $r_{i}=\frac{8}{7} q_{i}$ and choose $r_{0}$ and $r_{1}$ so that $r_{0}<\left(\frac{3}{4}-\varepsilon\right) r_{1}$, for some fixed small $\varepsilon>0$. Then

$$
r_{0}+\frac{1}{8} r_{i}<\left(\frac{7}{8}-\varepsilon\right) r_{i}, \quad i=1, \ldots
$$

which implies that

$$
V\left(B\left(r_{0}+\frac{1}{8} r_{i}\right)\right)<V\left(B\left(\left(\frac{7}{8}-\varepsilon\right) r_{i}\right)\right)
$$

and we are reduced to prove that

$$
\frac{V\left(B\left(r_{i}\right)\right)}{V\left(B\left(\frac{7}{8} r_{i}\right)\right)-V\left(B\left(\left(\frac{7}{8}-\varepsilon\right) r_{i}\right)\right)}
$$

is bounded.

Now, since $\lim \sup V\left(r_{i}\right) / r_{i}^{\alpha} \leqq c$, we see that, for all $\delta>0, V\left(r_{i}\right) \leqq c(1+\delta) r_{i}^{\alpha}$, for all but finitely many $r_{i}$ 's. Set $\bar{c}=c(1+\delta)$. Then, for all but finitely many $r_{i}$ 's,

$$
\frac{V\left(B\left(r_{i}\right)\right)}{V\left(B\left(\frac{7}{8} r_{i}\right)\right)-V\left(B\left(\left(\frac{7}{8}-\varepsilon\right) r_{i}\right)\right)} \leqq \frac{\bar{c} r_{i}^{\alpha}}{V\left(B\left(\frac{7}{8} r_{i}\right)\right)-\bar{c}\left(\frac{7}{8}-\varepsilon\right)^{\alpha} r_{i}^{\alpha}} .
$$


Thus, since $r_{i}=\frac{8}{7} q_{i}$,

$$
\begin{gathered}
\lim _{r_{i} \rightarrow \infty} \frac{V\left(B\left(r_{i}\right)\right)}{V\left(B\left(\frac{7}{8} r_{i}\right)\right)-V\left(B\left(\left(\frac{7}{8}-\varepsilon\right) r_{i}\right)\right)} \leqq \lim _{r_{i} \rightarrow \infty} \frac{\frac{\bar{c} r_{i}^{\alpha}}{\overline{\bar{c}(7 / 8)^{\alpha} r_{i}^{\alpha}}} \frac{\frac{1}{V\left(B\left((7 / 8) r_{i}\right)\right)}}{\bar{c}(7 / 8)^{\alpha} r_{i}^{\alpha}}-\frac{((7 / 8)-\varepsilon)^{\alpha}}{(7 / 8)^{\alpha}}}{\left(\overline{(7 / 8)^{\alpha}}\right.} \\
=\frac{1+\delta}{\frac{c}{\bar{c}}-\frac{((7 / 8)-\varepsilon)^{\alpha}}{(7 / 8)^{\alpha}}}=\frac{17 / 8)^{\alpha}-((7 / 8)-\varepsilon)^{\alpha}(1+\delta)}{}
\end{gathered}
$$

Since $\varepsilon$ is fixed, we can choose $\delta$ so small that the above denominator is strictly positive. It follows that, for this sequence, the limit of (2.6) exists and is finite. This proves our claim and the Lemma.

We want to thank Detang Zhou for kindly pointing us our mistake.

\section{References}

[1] H. AlenCar and M. Do CaRmo, Hypersurfaces of constant mean curvature with finite index and volume of polynomial growth. Arch. Math. 60, $489-493$ (1993).

Eingegangen am 9.12.1994

Anschrift der Autoren:

H. Alencar,

M. do Carmo

Instituto de Matemática Pura e Aplicada

Estrada Dona Castorina, 110

22460-320 Rio de Janeiro RJ

Brasil 